\title{
Non-hydrostatic effects on mountain wave breaking in directional shear flows
}

Article

Accepted Version

Guarino, M.-V. and Teixeira, M. A. C. (2017) Non-hydrostatic effects on mountain wave breaking in directional shear flows. Quarterly Journal of the Royal Meteorological Society, 143 (709). pp. 3291-3297. ISSN 1477-870X doi: https://doi.org/10.1002/qj.3157 Available at https://centaur.reading.ac.uk/72365/

It is advisable to refer to the publisher's version if you intend to cite from the work. See Guidance on citing.

Published version at: http://onlinelibrary.wiley.com/doi/10.1002/qj.3157/full

To link to this article DOI: http://dx.doi.org/10.1002/qj.3157

Publisher: Royal Meteorological Society

All outputs in CentAUR are protected by Intellectual Property Rights law, including copyright law. Copyright and IPR is retained by the creators or other copyright holders. Terms and conditions for use of this material are defined in the End User Agreement.

\section{www.reading.ac.uk/centaur}

\section{CentAUR}

Central Archive at the University of Reading 
Reading's research outputs online 


\title{
Non-hydrostatic effects on mountain wave breaking in directional shear flows
}

\author{
Maria-Vittoria Guarino*, Miguel A. C. Teixeira \\ Department of Meteorology, University of Reading, Reading, UK \\ ${ }^{*}$ Correspondence to: Maria Vittoria Guarino, Department of Meteorology, University of Reading, Earley Gate, PO Box 243, Reading \\ RG6 6BB, UK, E-mail: m.v.guarino@pgr.reading.ac.uk
}

\begin{abstract}
Mountain waves excited by narrow 3D orography are investigated using idealized numerical simulations of atmospheric flows with directional wind shear. The stability of these waves is compared with the stability of hydrostatic mountain waves. The focus is on understanding how wave breaking is modified via gravity wave-critical level interaction, when non-hydrostatic (dispersive) effects arise. The influence of nonhydrostatic effects on wave breaking appears to be a function of the intensity of the background shear, increasing the stability of the flow (inhibiting wave breaking) for weak wind shear, but decreasing it instead (enhancing wave breaking) for stronger wind shear.
\end{abstract}

Key Words: Mountain waves, Non-hydrostatic effects, Wave breaking, 3D mountains, Directional shear

Received...

\section{Introduction}

Non-hydrostatic mountain waves are primarily gravity waves excited by narrow orographic obstacles. Because of their dispersive nature, in a stratified atmosphere with constant background parameters, these waves are thought to be less likely to break than hydrostatic mountain waves (Laprise and Peltier 1989). The generation of approximately hydrostatic mountain waves is favoured either by a strongly stable atmospheric stratification (i.e. a large Brunt-Väisälä frequency $N_{0}$ ), a weak background wind $(U)$ or a broad orography (i.e. a large mountain half-width $a$ ), so that $N_{0} a / U \gg 1$. On the contrary, nonhydrostatic mountain waves are expected for a weak stratification, a strong background wind or a narrow orography, so that $N_{0} a / U \lesssim O(1)$. When $U$ and $N_{0}$ are constant, the character of the gravity waves launched by a mountain depends on the horizontal scale of the orography only (Holton and Hakim 2012). In the wide mountain limit, small horizontal wave-numbers dominate the wave spectrum: in this case the wave propagation and the energy transport are mostly vertical. In the narrow mountain limit, large horizontal wave-numbers dominate the wave spectrum and the wave propagation is partly horizontal, while the wave amplitude decays with height. Under these conditions the vertical velocity perturbations are not in hydrostatic balance.

Several studies investigated the dynamics of gravity waves in the two limits of vertically propagating and trapped lee waves, the latter being highly non-hydrostatic waves generated as a consequence of an increase in wind speed and/or a decrease in atmospheric stability with height (Scorer 1949, Durran 1986). Studies of hydrostatic mountain waves focused particularly on wave momentum deposition, wave breaking, and down-slope wind storms (e.g. McFarlane 1987, Clark and Peltier 1977, Bacmeister and Schoeberl 1989, Teixeira et al. 2004, Doyle and
Reynolds 2008); studies of trapped lee waves, on the other hand, mainly focused on lee wave rotors, surface drag, and orographic rain-bands (e.g. Vosper 2004, Kirshbaum et al. 2007, Stiperski and Grubišić 2011, Teixeira et al. 2013).

Fewer studies investigated the dynamics of non-trapped mountain waves when $N_{0} a / U=O(1)$ (e.g. Zängl 2003, Sachsperger et al. 2016). These are dispersive waves with short horizontal wavelengths partly able to propagate vertically and downstream of the orography that originated them. Because of destructive interferences taking place as waves propagate in the presence of dispersion, the wave amplitude decreases with distance away from their source (Nappo 2012). For a wind profile with directional shear, the dispersive nature of these waves may influence the occurrence of wave breaking via modification of the wave-critical level interaction, where critical levels correspond to those heights at which gravity waves amplify and may become unstable. Indeed, depending upon the strength of dispersion effects, less or more wave energy (as we shall see) may be available to be dissipated at critical levels.

The conditions under which hydrostatic mountain waves can break and initiate turbulence in the presence of directional wind shear were studied by Guarino et al. (2016). In that paper, idealized numerical simulations of atmospheric flows over orography were presented, and mountain wave breaking (within the troposphere) was diagnosed as a function of the orography elevation and wind shear required for its occurrence. The present study is an extension of Guarino et al. (2016), where the influence of non-hydrostatic effects on mid-tropospheric mountain wave breaking is analysed by taking into account the aforementioned dispersion effects, and also how the scaling of the velocity perturbations changes in non-hydrostatic flow.

In section 2, the set-up of numerical simulations and the method used to identify wave breaking within the simulation domain 
are briefly recalled. In section 3 , the stability of the flow in the transition from hydrostatic to non-hydrostatic mountain waves is discussed. In section 4 , the conclusions of this study are summarized.

\section{Methodology}

\subsection{Numerical simulations}

The idealized numerical simulations presented in this note use the same set-up as in Guarino et al. (2016), with the only differences being the mountain half-width, grid spacing and number of grid-points. The flow under consideration is adiabatic (i.e. no heat or moisture fluxes from the surface), inviscid (i.e. explicit diffusion not allowed anywhere, and no planetary boundary layer scheme used), and the Coriolis force is neglected. The inviscid approximation is motivated by the fact that we are focusing on the instabilities leading to the transition of a laminar flow to turbulence. Neglect of the Earth's rotation is justified by the small horizontal scale of the wave motion, even more than in the hydrostatic cases addressed by Guarino et al. (2016).

The simulations were performed using the WRF-ARW atmospheric model. The computational domain comprised 200 grid-points in both the $x$ and $y$-directions, with an isotropic grid spacing $\Delta x=\Delta y=500 \mathrm{~m}$. Such a high horizontal resolution was chosen to resolve properly the narrow mountains used in the simulations. The model vertical grid contained 200 eta levels (using a terrain-following hydrostatic-pressure coordinate), with spacing near the ground of $45 \mathrm{~m}$ and spacing at the top of the domain, $20 \mathrm{~km}$ above ground level (a.g.l.), of $450 \mathrm{~m}$. A 5 $\mathrm{km}$-deep absorbing sponge layer at the top of the domain was used to control wave reflection from the upper boundary. For those experiments performed with the strongest directional shear considered in this study, some adjustments were made to the model set-up to ensure numerical stability, namely: the top of the domain was raised to $30 \mathrm{~km}$, the depth of the absorbing layer was increased to $15 \mathrm{~km}$, and 400 eta vertical levels were used.

The model was initialized using an orography profile described by a 3D bell-shaped mountain:

$$
h(x, y)=\frac{H}{\left(\frac{x^{2}}{a^{2}}+\frac{y^{2}}{a^{2}}+1\right)^{3 / 2}}
$$

where $H$ is the maximum mountain height.

Non-hydrostatic mountain waves were generated by imposing $a=2.5 \mathrm{~km}, U=10 \mathrm{~m} \mathrm{~s}^{-1}$ and $N_{0}=0.01 \mathrm{~s}^{-1}$, so that $N_{0} a / U=$ 2.5. Note that this value of $N_{0} a / U$ leads to mountain waves that are moderately non-hydrostatic. The flow could be made even more non-hydrostatic by using a lower $N_{0} a / U$, however as $N_{0}$ and $U$ were chosen to be consistent with Guarino et al. (2016), and a smaller $a$ would correspond to unrealistically steep orography, $N_{0} a / U=2.5$ is near the smallest realistic value consistent with the chosen maximum value of $H$ (see below). Furthermore, strongly non-hydrostatic waves are limited in their vertical propagation (as will be discussed later) and hence are not so relevant to mid-tropospheric wave-breaking.

In the experiments, different degrees of flow non-linearity (associated with different wave amplitudes) were considered by using 5 values of the mountain height: $H=100 \mathrm{~m}, 200 \mathrm{~m}, 500 \mathrm{~m}$, $750 \mathrm{~m}, 1 \mathrm{~km}$. The vertical aspect ratio of the mountain $H / a$ varies in the range [0.04-0.4], and the non-dimensional mountain height defined in terms of $N_{0}$ and $U$ takes the values $N_{0} H / U=0.1,0.2$, $0.5,0.75,1$. For each orography configuration, 6 simulations using wind profiles with different intensities of the directional shear of the background flow were performed. This was implemented by changing the rate of wind turning with height $\beta$, which depends on the Richardson number of the background flow $\mathrm{Ri}_{i n}$ (for $N_{0}$ and $U$ constant with height) according to:

$$
u_{0}=U \cos (\beta z), \quad v_{0}=U \sin (\beta z),
$$

where $u_{0}$ and $v_{0}$ are the background wind components and $\beta=$ $N_{0} /\left(U \sqrt{\mathrm{Ri}_{\text {in }}}\right)$. More specifically, the values considered are: $\mathrm{Ri}_{\text {in }}$ $=16,8,4,2,1,0.5$, which correspond to $\beta \approx 14$ degrees $/ \mathrm{km}$, 20 degrees $/ \mathrm{km}, 31$ degrees $/ \mathrm{km}, 40$ degrees $/ \mathrm{km}, 57$ degrees $/ \mathrm{km}, 80$ degrees $/ \mathrm{km}$. Therefore, as $\mathrm{Ri}_{i n}$ decreases the rate of wind turning increases, resulting in flows with stronger directional wind shear.

As discussed by Guarino et al. (2016), the wind profile (2) can be viewed as a prototype of flows with directional shear. Directional shear is related to horizontal temperature gradients and thermal advection via thermal wind balance (Shutts and Gadian (1999) noted that a backing wind corresponds to cold advection). But that link will be ingnored here because the Earth's rotation does not affect the wave perturbations, and thus it may be neglected altogether, and the background wind treated as imposed. A flow in which the wind vector rotates with height by 180 degrees can be seen as an extension of the more often studied undirectional flow with a critical level (where $U=0$ ). Whereas such a critical level affects all the wave-numbers in the wave spectrum at the same height and the wave energy is dissipated in a thin layer, a continuous turning of the background wind with height creates a continuous distribution of critical levels in the vertical where the wave energy is absorbed into the background flow. Following, e.g., Shutts and Gadian (1999) and Teixeira et al. (2004), in the simulations presented here the wind vector rotates continuosly with height up to the model top (for mathematical convenience), but only the first 180 degrees of wind rotation are physically relevant and are analysed. The reader is directed to Guarino et al. (2016) for further discussion on the adopted wind profile.

\subsection{Wave breaking diagnosis}

The Richardson number of the flow including the wave perturbation, $\mathrm{Ri}_{\text {out }}$, was used to detect instability regions within the simulation domain. The three-dimensional $\mathrm{Ri}_{\text {out }}(x, y, z)$ field was computed at each grid-point using centred finite differences. Although Ri is notoriously sensitive to the depth of the layer on which the potential temperature and the wind gradients are calculated, the fairly high vertical resolution employed in the simulations guarantees that waves (and their overturning) are sufficiently well resolved everywhere in the simulation domain. Therefore, $\mathrm{Ri}_{\text {out }}$ is expected to provide reliable indications on the stability of the flow.

The $\operatorname{Ri}_{\text {out }}(x, y, z)$ field was used in the analysis of the results with the twofold aim of:

- Identifying the minimum $\mathrm{Ri}$ value in the field $\left(\mathrm{Ri}_{m i n}\right)$ for each experiment. When $\mathrm{Ri}_{\text {min }}$ is negative, flow overturning by wave breaking is assumed to occur in the simulation domain. $\mathrm{Ri}_{\text {min }}$ values are used to produce a regime diagram describing the wave breaking behaviour in the transition from linear to non-linear flows and from weak to strong directional shears.

- Delimiting regions where the wave propagation and breaking lead to the generation of dynamical $\left(\mathrm{Ri}_{\text {out }}<\right.$ $0.25)$ and/or convective $\left(\mathrm{Ri}_{\text {out }}<0\right)$ instabilities. Under these conditions the flow can potentially evolve into turbulence, hence these may be regarded as potentially turbulent regions.

Following Guarino et al. (2016), the $\mathrm{Ri}_{\text {out }}$ field was computed within a 'region of interest' delimited by upper, lower and lateral bounds. While for a more in-depth discussion and justification for 

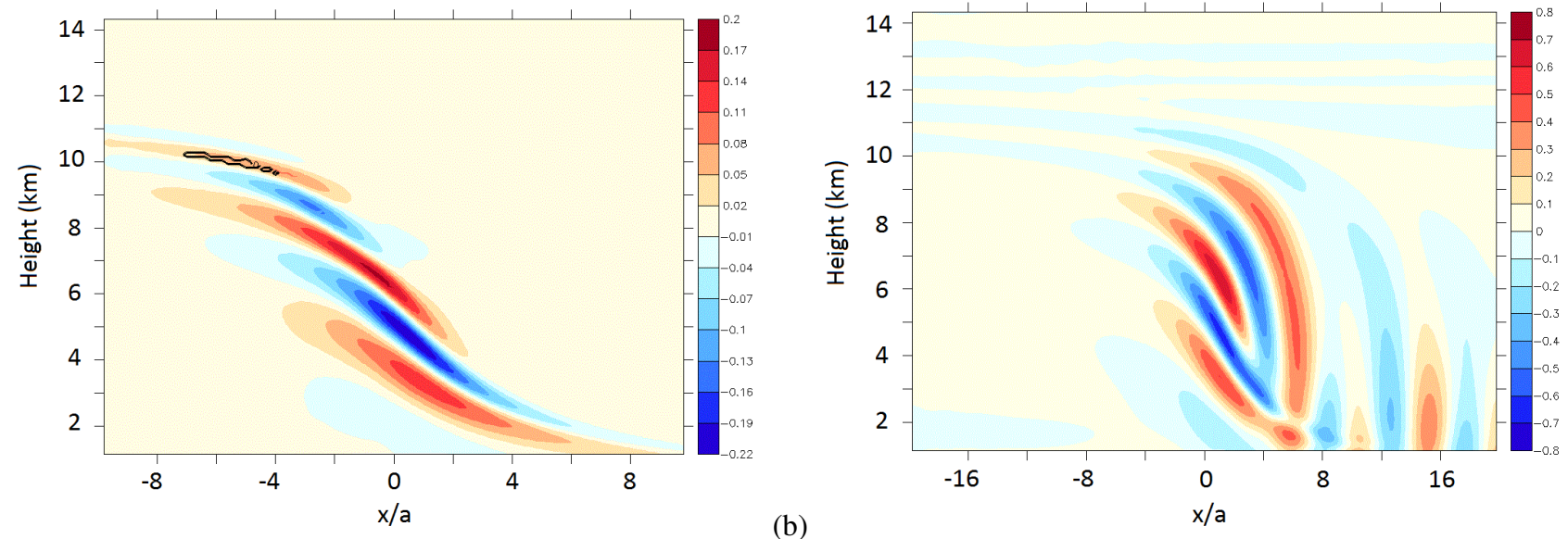

(a)

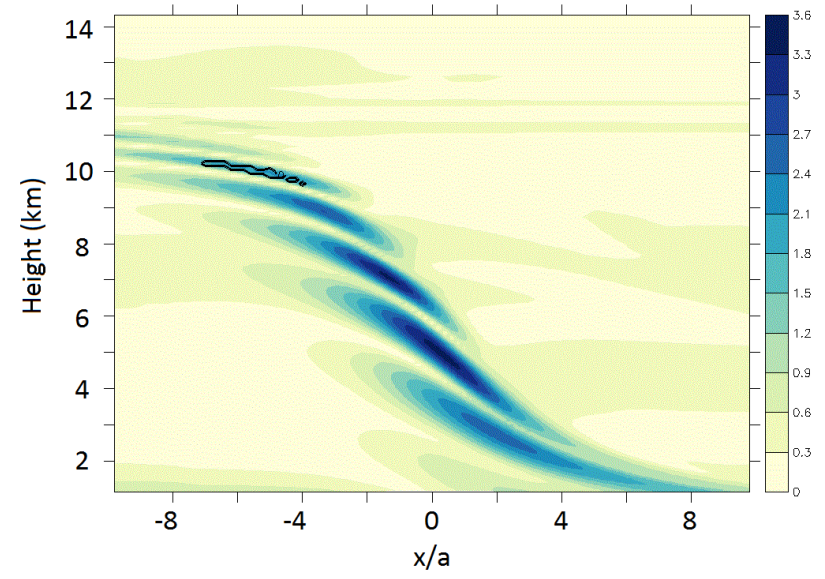

(b)

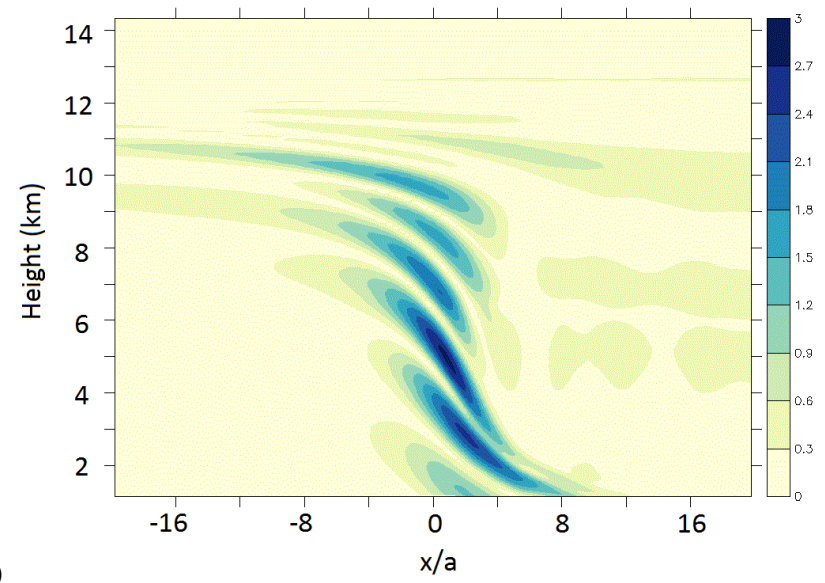

Figure 1. Vertical cross-sections of $w\left((\mathrm{a})\right.$ and (b)) and the magnitude of the wave horizontal velocity perturbation vector $\left(u^{\prime}, v^{\prime}\right)((\mathrm{c})$ and $(\mathrm{d}))$ at $\mathrm{Y} / a=+3.6$ for simulations with $\mathrm{Ri}_{i n}=16$ and $N_{0} H / U=1$. (a) and (c) show hydrostatic mountain waves $(a=10 \mathrm{~km})$, (b) and (d) show non-hydrostatic mountain waves $(a=2.5 \mathrm{~km})$. The black contours indicate regions where $\mathrm{Ri}_{\text {out }}<0$.

the choice of these bounds we refer to their article, we recall here that: the upper limit is $z \approx 14 \mathrm{~km}$, as it corresponds approximately to the height of the bottom of the sponge layer; the lower limit is $z \approx 1 \mathrm{~km}$, as in more realistic conditions this would correspond to the height of a fully developed Planetary Boundary Layer; the lateral limits represent a square region of $50 \times 50 \mathrm{~km}$ surrounding the mountain $(25 \mathrm{~km}$ to the east, west, north and south from the centre of the mountain). Note that while in Guarino et al. (2016) (where $a=10 \mathrm{~km}$ ) the region of interest had dimensions $100 \times 100$ $\mathrm{km}$, spanning from $-5 \mathrm{X} / a$ to $+5 \mathrm{X} / a$ along $x$ and from $-5 \mathrm{Y} / a$ to $+5 \mathrm{Y} / a$ along $y$ (where $\mathrm{X} / a$ and $\mathrm{Y} / a$ are distances relative to the mountain located at $(\mathrm{X}=0, \mathrm{Y}=0))$, for the simulations presented here (where $a=2.5 \mathrm{~km}$ ) the region of interest spans from $-10 \mathrm{X} / a$ to $+10 \mathrm{X} / a$ and from $-10 \mathrm{Y} / a$ to $+10 \mathrm{Y} / a$ instead. Considering larger relative distances is consistent with the dynamics of the more non-hydrostatic waves investigated in the present study, as these are expected to propagate laterally more than hydrostatic waves, resulting in more extended downwind disturbances (Nappo 2012). In the following section, the dynamics of non-hydrostatic mountain waves will be discussed in more detail.

\subsection{Non-hydrostatic mountain waves}

In its most general form, the Taylor-Goldstein equation for adiabatic, 3D, frictionless flows without rotation takes the form (Nappo 2012):

$$
\frac{d^{2} \widehat{w}}{d z^{2}}+\left[\frac{N_{0}^{2} k_{H}^{2}}{\left(k u_{0}+l v_{0}\right)^{2}}-\frac{k u_{0}^{\prime \prime}+l v_{0}^{\prime \prime}}{k u_{0}+l v_{0}}-k_{H}^{2}\right] \widehat{w}=0,
$$

where $\widehat{w}$ is the Fourier transform of the vertical velocity. For an extended range of applicability (beyond the Boussinesq approximation), this should be understood as a scaled vertical velocity, where the scaling factor accounts for the height dependency of the base-state density (Nappo 2012). $k$ and $l$ are the horizontal wave-numbers along the $x$ and $y$-directions, $k_{H}=$ $\sqrt{k^{2}+l^{2}}$ is the magnitude of the horizontal wave-number vector and the primes denote differentiation with respect to $z$.

In vertically sheared flows, the solution to (3) can be expressed as:

$$
\widehat{w}(k, l, z)=\widehat{w}(k, l, 0) \mathrm{e}^{i \int_{0}^{z} m(z) d z},
$$

where $m$ is the vertical wave-number. Equation (4) is subject to the lower boundary condition:

$$
\widehat{w}(k, l, 0)=i\left(k u_{0}+l v_{0}\right) \widehat{h}(k, l),
$$

where $\widehat{h}(k, l)$ is the Fourier transform of the terrain elevation $h(x, y)$. The last term in (3), involving $k_{H}^{2}$, accounts for the vertical acceleration of air parcels flowing across the mountain and is only present in the wave equation when this acceleration is important, thus accounting for non-hydrostatic effects.

By substituting (4) into (3) and adopting a zeroth-order WKB approximation, the vertical wave-number $m$ is defined as:

$$
m=\left[\frac{N_{0}^{2} k_{H}^{2}}{\left(k u_{0}+l v_{0}\right)^{2}}-k_{H}^{2}\right]^{1 / 2} .
$$

In the hydrostatic limit $\left(N_{0} a / U \gg 1\right), k_{H}$ is negligible so that $m=N_{0} k_{H} /\left(k u_{0}+l v_{0}\right)$. In the strongly non-hydrostatic limit $\left(N_{0} a / U \ll 1\right)$, buoyancy forces are unimportant compared to $k_{H}$ and the vertical wave-number is imaginary: $m=i k_{H}$. In the first case, the wave propagation is vertical and governed by 


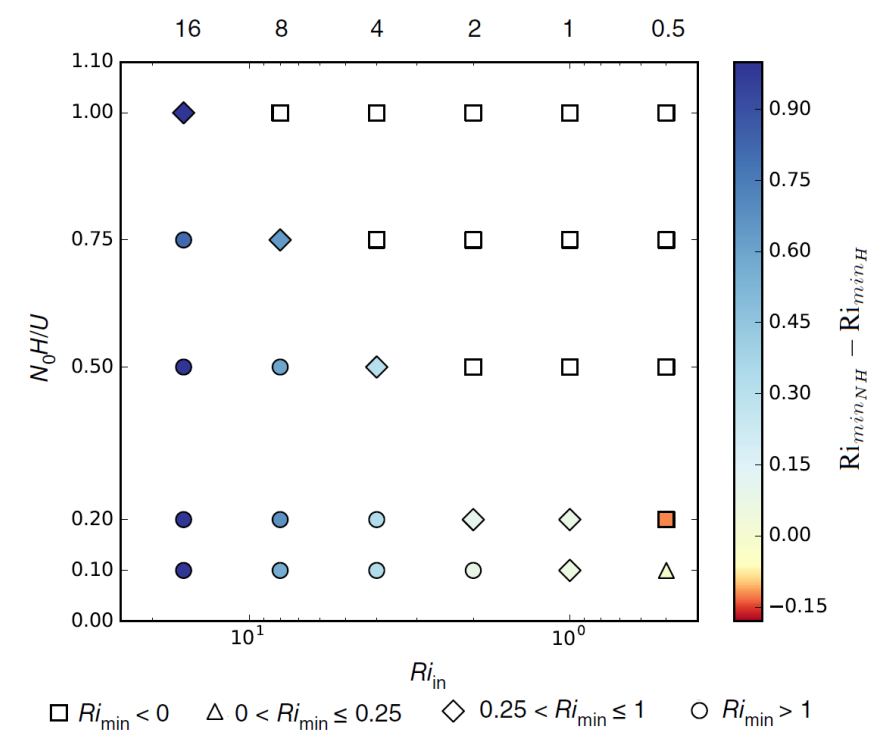

Figure 2. Regime diagram describing flow stability and likeliness of wave breaking as a function of $N_{0} H / U$ and $\mathrm{Ri}_{\text {in }}$. The $\mathrm{Ri}_{\text {in }}$ values use a logarithmic scale, however the actual $\mathrm{Ri}_{\text {in }}$ values considered are shown on the upper horizontal axis.

buoyancy forces. In the second case, the generation of verticallypropagating gravity waves is inhibited and disturbances propagate in the horizontal direction as evanescent waves trapped at the lower boundary. The present study stands somewhere in between these two limit cases, as non-hydrostatic (but still verticallypropagating) gravity waves, with a dispersion relationship given by (6), are investigated.

According to (6), the dependence of the vertical wave-number on the horizontal wave-number introduces dispersion effects to the gravity wave dynamics in addition to those associated with the three-dimensionality of the flow. Indeed, because of the presence of the term $\left(k u_{0}+l v_{0}\right)$ in (6), 3D hydrostatic mountain waves are already dispersive. This type of dispersion (often termed 'directional dispersion') causes the wave energy to spread along a parabola that widens with height, causing the waves to weaken as they propagate upwards (Smith 1980). The dispersion added by the non-hydrostatic term in (6) decreases the total wave energy density by allowing horizontal wave propagation and downstream spatial spreading of the wave packet, as can be deduced from group velocity arguments (Nappo 2012).

\section{Results and discussion}

\subsection{Non-hydrostatic effects on wave breaking}

Figure 1 shows vertical cross-sections of $w$ and the horizontal velocity perturbation $\left(u^{\prime}, v^{\prime}\right)$ for two simulations with $\mathrm{Ri}_{i n}=16$ and $H=1 \mathrm{~km}$ in the case of hydrostatic (Figure 1(a) and 1(c)) and non-hydrostatic (Figure 1(b) and 1(d)) mountain waves. The cross-sections were taken at $\mathrm{Y} / a=+3.6$ where, in the hydrostatic case, wave breaking occurs. The black contours correspond to $\mathrm{Ri}_{\text {out }}<0$. In Figure 1(a) and 1(c), hydrostatic waves propagate vertically up to $z \approx 12 \mathrm{~km}$. For non-hydrostatic mountain waves, a roughly similar flow behaviour is observed, but in Figure 1(b) the wave propagation shows a significant downstream component, highlighting the dispersive nature of the disturbance. While hydrostatic waves break at $z \approx 10 \mathrm{~km}$ (where $\mathrm{Ri}_{\text {out }}<0$ ), the breaking region is absent in Figure $1(\mathrm{~b})$ and $1(\mathrm{~d})$. Since in directional shear flows the existence/location of critical levels depend on the relative orientation of the background wind vector and the horizontal wave number vector, and the two simulations were initialized with the same wind profile and orography, we can assume that the same directional critical levels exist in both flow configurations. However, for non-hydrostatic mountain waves the wave dispersion presumably makes the wave energy decay faster, producing destructive interferences as the waves propagate. As a consequence, for wave packets approaching the critical level at $z \approx 10 \mathrm{~km}$, the wave energy is not concentrated enough to cause flow overturning and wave breaking. As a further confirmation, while $\mathrm{Ri}_{\text {min }}$ in the hydrostatic waves occurs at $z \approx 10 \mathrm{~km}$ $\left(\mathrm{Ri}_{\text {min }_{H}}=-23\right)$, for non-hydrostatic waves $\mathrm{Ri}_{\text {min }}$ occurs at much lower altitudes $z \approx 3 \mathrm{~km}\left(\mathrm{Ri}_{\text {min }_{N H}}=0.75\right)$ where, plausibly, dispersive effects are less effective. Indeed, the cumulative effect of dispersion becomes stronger in the far-field, after waves have propagated over long distances. Besides dispersive effects, hydrostatic and non-hydrostatic mountain waves differ in the scaling of key flow parameters (namely the horizontal velocity perturbations), as is discussed below.

In Figure 1(b) non-hydrostatic mountain waves exhibit near the surface a vertical velocity which is 4 times larger than the vertical velocity for hydrostatic waves (the maximum on the $w$ scale is 0.2 in Figure 1(a) and 0.8 in Figure 1(b)). This can be explained via the bottom boundary condition (5), from which $w$ scales as $\frac{U H}{a}$ (both for hydrostatic and non-hydrostatic waves). Because the $a$ used in the non-hydrostatic simulations $\left(a_{N H}=2.5 \mathrm{~km}\right)$ is 4 times smaller than the one used in the hydrostatic simulations $\left(a_{H}=10\right.$ $\mathrm{km}$ ), the corresponding $w$ field in Figure 1 has a magnitude that is 4 times larger than the one for hydrostatic waves $\left(w_{N H} \approx 4 w_{H}\right)$.

Unlike the scaling for $w$, the scaling predicted by linear theory for the horizontal velocity perturbations must be distinguished between the hydrostatic and non-hydrostatic cases. From mass conservation, and for the flow parameters considered here, it can be shown that while in the hydrostatic case this scaling is $v_{H}^{\prime} \sim u_{H}^{\prime} \sim \frac{N a_{H}}{U} w_{H} \approx 10 w_{H}$, in the strongly non-hydrostatic case $v_{N H}^{\prime} \sim u_{N H}^{\prime} \sim w_{N H} \approx 4 w_{H}$.

In Figure 1(c) the maximum on the $\left|\left(u^{\prime}, v^{\prime}\right)\right|$ scale is $3.6 \mathrm{~m} \mathrm{~s}^{-1}$, and in Figure 1(d) it is $3 \mathrm{~m} \mathrm{~s}^{-1}$. Thus, the flow cross-sections show that the simulated horizontal velocity perturbation is larger than the hydrostatic estimate of $10 w_{H}=10 \times 0.2 \mathrm{~m} \mathrm{~s}^{-1}=2 \mathrm{~m} \mathrm{~s}^{-1}$ for the hydrostatic case (Figure 1(c)), and much larger than the strongly non-hydrostatic estimate of $4 w_{H}=4 \times 0.2 \mathrm{~m} \mathrm{~s}^{-1}=0.8$ $\mathrm{m} \mathrm{s}^{-1}$ for the non-hydrostatic case (Figure 1(d)). Note, however, that influence (albeit weak) from the non-hydrostatic scaling is still noticeable, as the horizontal velocity perturbation is slightly smaller than the one in Figure 1(c).

It should be kept in mind that the scalings of the horizontal velocity perturbations described above are strictly valid for linear flows, but the flows in Figure 1 are highly non-linear (as testified by the presence of wave breaking). Although departures from the estimated values are expected, the similar magnitude of $\left(u^{\prime}, v^{\prime}\right)$ in the hydrostatic and non-hydrostatic cases suggests that the hydrostatic scaling still applies more closely to the nonhydrostatic case than the strongly non-hydrostatic scaling (which is only valid when the effects of stratification become vanishingly weak). This is not surprising because, as discussed in section 2.1, the mountain waves considered in this study are only moderately non-hydrostatic.

The interplay between the scaling of the velocity perturbations and the wave dispersion effects is not trivial, as both these mechanisms contribute to determine the wave amplitude and, thus, the likeliness of wave breaking. It should be noted, additionally, that the scalings presented above are applicable locally near the surface (they use the orography width as a horizontal length scale, for example), whereas the effect of dispersion is intrinsically related to the propagation of the waves. The role of each of these two mechanisms in wave breaking is discussed in the following section. 


\subsection{A regime diagram for non-hydrostatic mountain waves}

In Figure 2, a regime diagram describing the stability of the flow as a function of its non-linearity (quantified through $N_{0} H / U$ ) and directional shear intensity (quantified through $\mathrm{Ri}_{i n}$ ) is presented. This regime diagram is directly comparable with the regime diagram for hydrostatic waves presented in Figure 3 of Guarino et al. (2016), and depicts stability conditions under which a flow can become turbulent, although turbulence itself is not modelled in the experiments. The likeliness of wave breaking is diagnosed by using $\mathrm{Ri}_{\min _{N H}}$, calculated for each numerical simulation, after the surface drag stabilizes to a constant value. To generate the regime diagram, time-series of $\mathrm{Ri}_{\min _{N H}}$ over a period of seven hours were analyzed. In the absence of wave breaking, the perturbation pattern associated with mountain waves is stationary in time. Thus, for those simulations where wave breaking does not occur ( $\mathrm{Ri}_{\min _{N H}}$ positive) hourly $\mathrm{Ri}_{\min _{N H}}$ values were found to be nearly constant. By contrast, in those simulations where wave breaking occurs $\left(\mathrm{Ri}_{\min _{N H}}\right.$ negative) hourly $\mathrm{Ri}_{\min _{N H}}$ values varied in time but remained negative. Four $\mathrm{Ri}_{\min _{N H}}$ categories were chosen, in accordance with the background literature, to represent the stability of the flow: $\mathrm{Ri}_{\min _{N H}}<0$ (squares) indicating convective instability due to wave breaking events (category 1 ), $0<\mathrm{Ri}_{\text {min }_{N H}} \leq 0.25$ (triangles) indicating dynamic instability, which is potentially an index of turbulence (category 2), $0.25<\mathrm{Ri}_{\min _{N H}} \leq 1$ (diamonds) indicating a flow having enough kinetic energy available for turbulent mixing (category 3 ), and $\mathrm{Ri}_{\text {min }_{N H}}>1$ (circles), indicating non-turbulent flow where no wave breaking events occur (category 4 ).

The colours in Figure 2 denote the difference between the $\mathrm{Ri}_{\min _{N H}}$ values obtained from the non-hydrostatic simulations and the corresponding $\mathrm{Ri}_{\text {min }_{H}}$ values from Guarino et al. (2016). This allows us to visualize in which areas of the regime diagram the non-hydrostatic effects: stabilize the flow (positive differences), increase its instability (negative differences), or leave it unaltered (differences near zero). Because the information about the changes in flow stability is given by the sign of the quantity $\left(\mathrm{Ri}_{\min _{N H}}-\mathrm{Ri}_{\min _{H}}\right)$ and because positive differences were found to be larger than negative ones, the scale is bounded between -0.2 and 1 . Also note that when $\mathrm{Ri}_{\text {min }}$ is negative in both non-hydrostatic and hydrostatic conditions, the value of $\left(\mathrm{Ri}_{\min _{N H}}-\mathrm{Ri}_{\text {min }_{H}}\right.$ ) is ignored (white squares in Figure 2). This is because, once the Richardson number drops below zero, any negative value has roughly the same meaning in terms of flow instability, and large differences that might occur would convey a misleading idea about their physical significance.

Just as in the hydrostatic waves case, wave breaking is more likely to happen for flows with low $\mathrm{Ri}_{i n}$ and high $N_{0} H / U$, which is expected physically. However, while on the left-hand side of the regime diagram $\left(\mathrm{Ri}_{i n}=16,8\right)$ the non-hydrostatic effects tend to stabilize the flow $\left(\mathrm{Ri}_{\min _{N H}}>\mathrm{Ri}_{\text {min }_{H}}\right)$, on the righthand side $\left(\mathrm{Ri}_{i n}=0.5\right)$ the instability of the flow becomes stronger $\left(\mathrm{Ri}_{\min _{N H}}<\mathrm{Ri}_{\text {min }_{H}}\right)$.

Details about the changes in flow stability observed in the transition from hydrostatic to non-hydrostatic waves are summarized in Table 1. Of particular interest are the changes in flow stability for the cases where:

- $\mathrm{Ri}_{\text {in }}=16$ and $N_{0} H / U=1$. In this case, non-hydrostatic mountain waves are not able to perturb the background flow as strongly as hydrostatic waves $\left(\mathrm{Ri}_{\min _{N H}}>\mathrm{Ri}_{\text {min }_{H}}\right)$ and the wave breaking that was observed in the hydrostatic case no longer occurs;

- $\mathrm{Ri}_{i n}=0.5$ and $N_{0} H / U=0.2$. Here $\mathrm{Ri}_{\min _{N H}}<\mathrm{Ri}_{\text {min }_{H}}$, and $\mathrm{Ri}_{\text {min }_{N H}}$ is negative. Hence, non-hydrostatic mountain waves break leading to overturning regions not present in the hydrostatic simulations.
On the left-hand side of the regime diagram, for the largest values of $\mathrm{R}_{i n}$, the increased stability of the flow can be explained by the dispersion effects discussed in the previous section and illustrated in Figure 1(b). On the right-hand side, for the lowest values of $\mathrm{Ri}_{\text {in }}$, the larger instability of the flow is probably a result of the larger value of $w$ in the non-hydrostatic simulations, the lesser importance of dispersion effects in strong directional shear, and nonlinear effects, as will be discussed further below.

As noted by Guarino et al. (2016), for wind profiles with a fast rate of wind turning with height, a high density of critical levels exists at low levels in the atmosphere. Directional critical levels for a particular wave-number in the wave spectrum are defined as the heights where the background wind vector is perpendicular to the horizontal wave number vector, so that in (6) $k u_{0}+l v_{0}=0$ and consequently $m \rightarrow \infty$. Figure 3 shows all the grid-points where $\mathrm{Ri}_{\text {out }}<0.25$ for the numerical simulations with $N_{0} H / U$ $=1$. The $\mathrm{Ri}_{\text {out }}<0.25$ field corresponds to dynamical instability regions, which may contain smaller regions where the flow overturns $\left(\mathrm{Ri}_{\text {out }}<0\right)$. In the strongest shear flow considered in this study, where $\mathrm{Ri}_{\text {in }}=0.5$, the wind rotates by 180 degrees in the first $2.5 \mathrm{~km}$ of the atmosphere, hence the condition $k u_{0}+l v_{0}=0$ occurs at least once for each wave-number in the wave spectrum over this depth. Waves are likely to break at the lowest critical level they encounter, therefore the majority of the wave energy is expected to dissipate by wave breaking within the first $2.5 \mathrm{~km}$ of the atmosphere. A similar kind of behaviour is observed for the wind profiles with larger $\mathrm{Ri}_{\text {min }}$ (weaker shear flows), where the instability regions extend across deeper atmospheric layers. Because wave-dispersion is a function of the distance over which waves propagate (i.e. it becomes cumulatively stronger at large vertical or horizontal - distances from the mountain), dispersive effects are stronger for waves travelling up to $12 \mathrm{~km}\left(\mathrm{Ri}_{\text {in }}=16,8\right.$, 4), and much weaker for waves travelling for less than $2.5 \mathrm{~km}$ before they reach their critical levels (where, incidentally, they become almost perfectly hydrostatic) and break $\left(\mathrm{Ri}_{\text {in }}=0.5\right)$. Also note that in Figure 3(a), for $\mathrm{Ri}_{i n}=8$, instabilities occur at low levels, and the higher-altitude overturning regions observed in the hydrostatic case (see Guarino et al. (2016) Figure 6(b)) are absent, confirming that dispersion limits the wave amplitude at higher altitudes and, thus, selectively influences the wave-critical level interaction.

As discussed in subsection 3.1, while $w_{N H} \approx 4 w_{H}$ in the simulations, $u_{N H}^{\prime}$ is only slightly smaller than $u_{H}^{\prime}$ (see also Figure 1(c) and 1(d)). This leads to a higher total wave kinetic energy, enhancing wave amplitude and likeliness of breaking at critical levels (where $w \rightarrow 0$ and $\left(u^{\prime}, v^{\prime}\right) \rightarrow \infty$ according to linear theory (Shutts 1998)). As a consequence, at the same critical level, the wave amplitude is expected to increase more markedly for non-hydrostatic than for hydrostatic flow. Thus, in the absence of significant dispersion, non-hydrostatic mountain waves may become unstable and break where hydrostatic mountain waves do not. This probably explains why in Figure 2, when $\mathrm{Ri}_{\text {in }}=$ 0.5 and $N_{0} H / U=0.1, \mathrm{Ri}_{\text {min }_{N H}}<\mathrm{Ri}_{\text {min }_{H}}$ and the decrease in $\mathrm{Ri}_{\text {min }_{N H}}$ (albeit small) causes the flow to shift from category 3 (for hydrostatic waves) to category $2^{*}$. When the mountain height increases even further, so that $N_{0} H / U=0.2$, the wave amplitude is large enough to cause flow overturning and wave breaking. For larger $N_{0} H / U$ no differences are observed, as wave breaking is expected also in the hydrostatic regime. An alternative, but probably equivalent, interpretation of this behaviour is that wave non-linearity is not only controlled by $N_{0} H / U$, but also

\footnotetext{
${ }^{*}$ Note that this is the only flow in the regime diagram belonging to category 2 , which seems to be under-represented. While this could be a consequence of a relatively sparse sampling of parameter space, a steady state mountain wave field with $0<\mathrm{Ri}$ $<0.25$ is nevertheless difficult to attain because of the development of dynamical instability. See Guarino et al. (2016) for further discussion.
} 
Table 1. Summary of the results in Figure 2, including details about changes in flow stability observed in the transition from hydrostatic to non-hydrostatic waves, namely: flow type $\left(\mathrm{Ri}_{i n}\right.$ and $\left.N_{0} H / U\right), \mathrm{Ri}_{\text {min }}$ for hydrostatic and non-hydrostatic mountain waves, observed changes in flow stability and corresponding flow regimes. For any parameter combinations not shown, the flow class remained unchanged.

\begin{tabular}{lllll}
\hline Flow type & $\mathrm{Ri}_{\min _{H}}$ & $\mathrm{Ri}_{\min _{N H}}$ & Stability & Flow regime \\
\hline $\mathrm{Ri}_{\text {in }}=16, N_{0} H / U=0.5,0.75$ & {$[0.25,1]$} & $>1$ & increasing & may evolve into turbulence $\rightarrow$ stable \\
$\mathrm{Ri}_{i n}=16, N_{0} H / U=1$ & $<0$ & {$[0.25,1]$} & increasing & convective instability $\rightarrow$ may evolve into turbulence \\
$\mathrm{Ri}_{\text {in }}=8, N_{0} H / U=0.5$ & {$[0.25,1]$} & $>1$ & increasing & may evolve into turbulence $\rightarrow$ stable \\
$\mathrm{Ri}_{i n}=8, N_{0} H / U=0.75$ & {$[0,0.25]$} & {$[0.25,1]$} & increasing & dynamical instability $\rightarrow$ may evolve into turbulence \\
$\mathrm{Ri}_{i n}=0.5, N_{0} H / U=0.1$ & {$[0.25,1]$} & {$[0,0.25]$} & decreasing & may evolve into turbulence $\rightarrow$ dynamical instability \\
$\mathrm{Ri}_{i n}=0.5, N_{0} H / U=0.2$ & {$[0,0.25]$} & $<0$ & decreasing & dynamical instability $\rightarrow$ convective instability \\
\hline
\end{tabular}

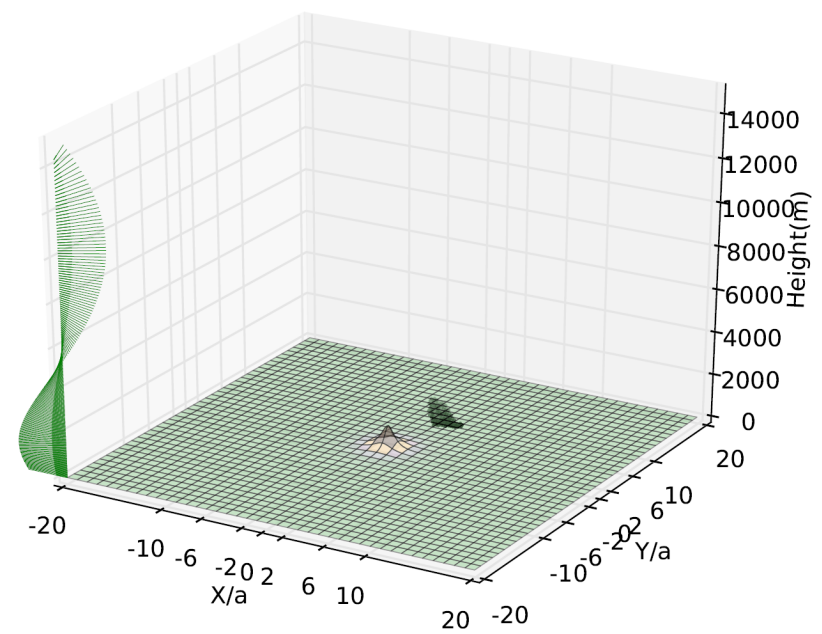

(a)

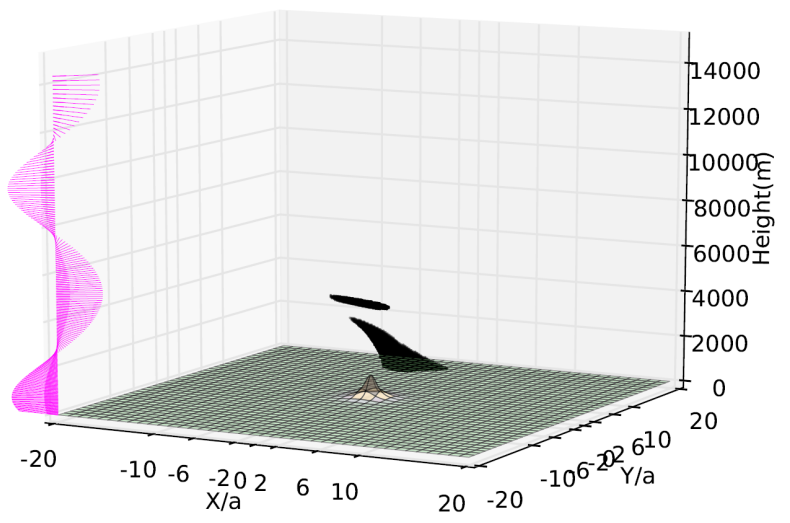

(b)

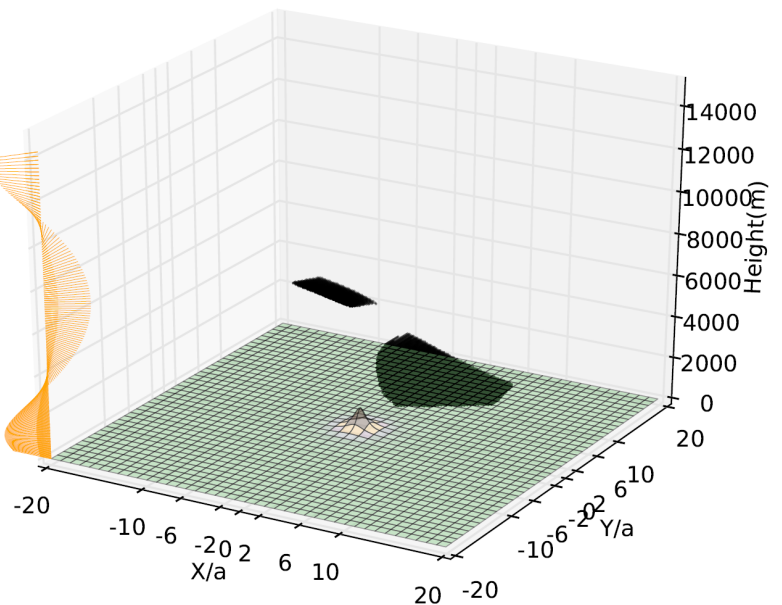

(d)

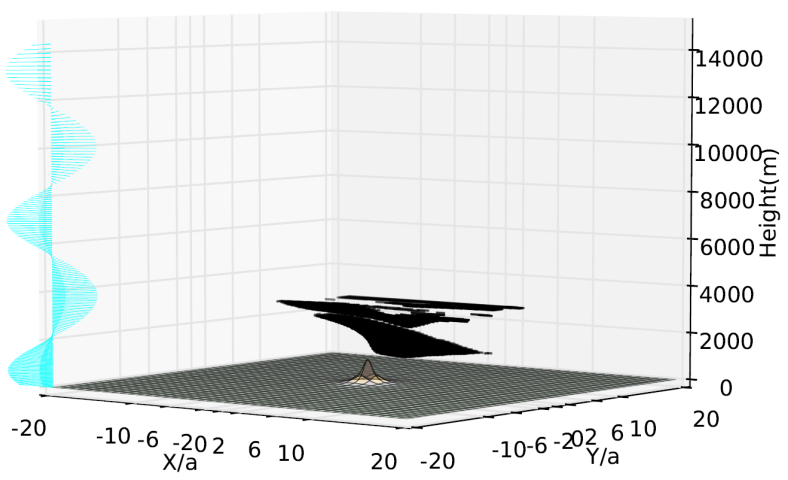

(c)

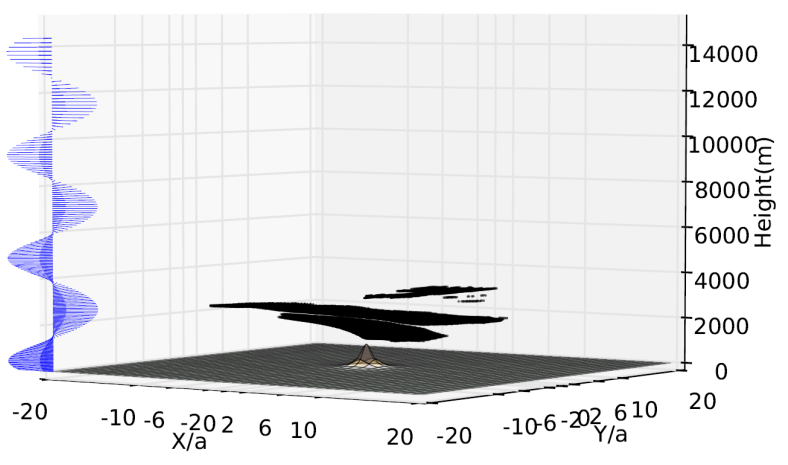

(e)

Figure 3. 3D perspective of the flow showing every point in the computational domain where $\mathrm{Ri}_{\text {out }}<0.25$. All panels refer to simulations performed with $N_{0} H / U=1$ and different wind shear intensities: (a) $\mathrm{Ri}_{\text {in }}=8$, (b) $\mathrm{Ri}_{\text {in }}=4$, (c) $\mathrm{Ri}_{\text {in }}=2$, (d) $\mathrm{Ri}_{\text {in }}=1$, (e) $\mathrm{Ri}_{\text {in }}=0.5$. On the left-hand side of each plot, a representation of the wind profile used in the simulations is shown. The wind profile has a helical shape corresponding to a wind that is westerly at the surface and rotates anticlockwise as $z$ increases.

increasingly by $H / a$ as the flow becomes more non-hydrostatic. Since $H / a$ is larger by a factor of 4 here than in the hydrostatic simulations of Guarino et al. (2016), this may explain why wave breaking can become more likely despite the contrary effect of wave dispersion.

To conclude, the stability of flows where $\mathrm{Ri}_{\text {in }}=4$ and 2 , in the centre portion of the regime diagram in Figure 2, is not significantly affected by non-hydrostatic effects. The differences $\left(\mathrm{Ri}_{\text {min }_{N H}}-\mathrm{Ri}_{\text {min }_{H}}\right)$ there are close to zero and no changes in flow stability (i.e. $\mathrm{Ri}_{\text {min }}$ category) are observed in the transition from hydrostatic to non-hydrostatic mountain waves. This can be interpreted as resulting from a balance between the two mechanisms acting to decrease (dispersion) or enhance (orography slope) the wave amplitude, as described above. 


\section{Summary and conclusions}

In this note, flow stability changes in the transition from hydrostatic to non-hydrostatic gravity waves generated over a narrow axisymmetric mountain in the presence of directional wind shear has been investigated. In particular, the analysis focused on understanding how non-hydrostatic effects can prevent or favour wave breaking relative to hydrostatic flow. A set of numerical simulations were performed extending Guarino et al. (2016), where the conditions for mountain wave breaking were diagnosed as a function of the orography elevation and wind shear, quantified by the dimensionless mountain height $N_{0} H / U$ and the Richardson number of the background flow $\mathrm{Ri}_{i n}$, respectively. The orographic gravity waves considered in this study are sufficiently affected by non-hydrostatic effects $\left(N_{0} a / U=O(1)\right)$ for dispersion and horizontal propagation to become important, but still too far from the strongly non-hydrostatic limit $\left(N_{0} a / U \ll\right.$ 1) for vertical propagation to be strongly inhibited.

The main conclusions from this study can be summarized as follows:

- For weaker shear flows, non-hydrostatic effects increase the stability of the flow. This is a consequence of the additional wave dispersion occurring in this case, which acts to decrease the wave amplitude when waves travel over long distances in the vertical before they reach a critical level and break.

- For stronger shear flows, non-hydrostatic effects decrease the stability of the flow. Here, dispersion effects are weaker because waves only propagate over short vertical distances before breaking, and additional instability seems to be caused primarily by the higher slope of the orography.

- The transition from stabilizing to de-stabilizing nonhydrostatic effects occurs gradually, and appears to be a function of the intensity of the directional shear of the background flow, perhaps because this controls the distance over which dispersion effects can act before the waves break.

In summary, although because of their dispersive nature nonhydrostatic mountain waves are generally believed to be less likely to break (Laprise and Peltier 1989), in the presence of directional wind shear this seems to be only partially true. In the numerical simulations presented in this study, flow overturning was detected over lower mountains $\left(N_{0} H / U=0.2\right.$, or $\left.H=200 \mathrm{~m}\right)$ than in the corresponding hydrostatic wave case, where the lowest $N_{0} H / U$ value associated with wave breaking was 0.5 , or $H=$ $500 \mathrm{~m}$ (Guarino et al. 2016). Admittedly, a more comprehensive exploration of parameter space would be necessary to ascertain the robustness of this finding.

The present results could be relevant to improve mountain wave breaking and turbulence diagnostics, for example, used in ClearAir Turbulence forecasts for aviation.

\section{Acknowledgements}

M.V.G. and M.A.C.T. acknowledge the financial support of the European Commission under Marie Curie Career Integration Grant GLIMFLO, contract PCIG13-GA-2013-618016.

\section{References}

Bacmeister JT, Schoeberl MR. 1989. Breakdown of vertically propagating two-dimensional gravity waves forced by orography. Journal of the Atmospheric Sciences 46: 2109-2134.

Clark T, Peltier W. 1977. On the evolution and stability of finite-amplitude mountain waves. Journal of the Atmospheric Sciences 34: 1715-1730.
Doyle JD, Reynolds CA. 2008. Implications of regime transitions for mountain-wave-breaking predictability. Monthly Weather Review 136: 5211-5223.

Durran DR. 1986. Another look at downslope windstorms. part i: The development of analogs to supercritical flow in an infinitely deep, continuously stratified fluid. Journal of the Atmospheric Sciences 43: 25272543.

Guarino MV, Teixeira MA, Ambaum MH. 2016. Turbulence generation by mountain wave breaking in flows with directional wind shear. $Q$. J. R. Meteorol. Soc. 142: 2715-2726.

Holton JR, Hakim GJ. 2012. An introduction to dynamic meteorology. Academic press.

Kirshbaum DJ, Bryan GH, Rotunno R, Durran DR. 2007. The triggering of orographic rainbands by small-scale topography. Journal of the atmospheric sciences 64: 1530-1549.

Laprise R, Peltier WR. 1989. On the structural characteristics of steady finiteamplitude mountain waves over bell-shaped topography. Journal of the atmospheric sciences 46: 586-595.

McFarlane NA. 1987. The effect of orographically excited gravity wave drag on the general circulation of the lower stratosphere and troposphere. $J$. Atmos. Sci. 44: 1775-1800.

Nappo CJ. 2012. An introduction to atmospheric gravity waves, 2nd ed. Academic Press.

Sachsperger J, Serafin S, Grubišić V. 2016. Dynamics of rotor formation in uniformly stratified two-dimensional flow over a mountain. Quarterly Journal of the Royal Meteorological Society 142: 1201-1212.

Scorer R. 1949. Theory of waves in the lee of mountains. Quarterly Journal of the Royal Meteorological Society 75: 41-56.

Shutts GJ. 1998. Stationary gravity-wave structure in flows with directional wind shear. Q. J. R. Meteorol. Soc. 124: 1421-1442.

Shutts GJ, Gadian A. 1999. Numerical simulations of orographic gravity waves in flows which back with height. Q. J. R. Meteorol. Soc. 125: 27432765 .

Smith RB. 1980. Linear theory of stratified hydrostatic flow past an isolated mountain. Tellus 32: 348-364.

Stiperski I, Grubišić V. 2011. Trapped lee wave interference in the presence of surface friction. Journal of the Atmospheric Sciences 68: 918-936.

Teixeira MA, Argaín JL, Miranda PM. 2013. Orographic drag associated with lee waves trapped at an inversion. Journal of the Atmospheric Sciences 70: 2930-2947.

Teixeira MAC, Miranda PMA. 2009. On the momentum fluxes associated with mountain waves in directionally sheared flows. J. Atmos. Sci. 66: 34193433.

Teixeira MAC, Miranda PMA, Valente MA. 2004. An analytical model of mountain wave drag for wind profiles withshear and curvature. J. Atmos. Sci. 61: 1040-1054.

Vosper S. 2004. Inversion effects on mountain lee waves. Quarterly Journal of the Royal Meteorological Society 130: 1723-1748.

Zängl G. 2003. Orographic gravity waves close to the nonhydrostatic limit of vertical propagation. Journal of the atmospheric sciences 60: 2045-2063. 